\title{
Acquisition of Rainfall in Ungauged Basins: a Study of Rainfall Distribution Heterogeneity Based on a New Method
}

\section{Ye Zhao}

Wuhan University

Xiang zhang ( $\nabla$ zhangxiang@whu.edu.cn )

Wuhan University School of Water Resources and Hydropower Engineering

feng xiong

Changjiang Water Resources Commission

Shuying Liu

Wuhan University

yao wang

Wuhan University

changmei liang

hubei water resources research institude

\section{Research Article}

Keywords: Rainfall, Proper orthogonal decomposition, Spatiotemporal variations, Ungauged basin, Interpolation

Posted Date: January 11th, 2022

DOI: https://doi.org/10.21203/rs.3.rs-1237636/v1

License: (c) (i) This work is licensed under a Creative Commons Attribution 4.0 International License. Read Full License 


\title{
Acquisition of rainfall in ungauged basins: a study of rainfall distribution heterogeneity based on a new method
}

\author{
Ye Zhao $^{\mathrm{a}, \mathrm{b}}$ Xiang Zhanga,b* Feng Xiong ${ }^{\mathrm{c}} \quad$ Shuying Liu $^{\mathrm{a}, \mathrm{b}} \quad$ Yao Wanga \\ Changmei Liangd \\ a. State Key Laboratory of Water Resources and Hydropower Engineering Science, Wuhan
}

University, 430072, Wuhan, China

b. Hubei Key Lab of Water System Science for Sponge City Construction, 430072, Wuhan, China

c. Bureau of Hydrology, Changiiang Water Resources Commission, 430010, Wuhan, China

d. Hubei Water Resources Research Institute, Wuhan, 430070, China

\begin{abstract}
High-density precipitation data is always desired to capture the heterogeneity of precipitation to accurately describe the components of the hydrological cycle. However, equipping and maintaining a high-density rain gauge network involves high costs, and the existing rain gauges are often unable to meet the density requirements. The objective of this study is to provide a new method to analyze the spatiotemporal variability of the precipitation field and to solve the problem of insufficient site density. To this end, the Proper Orthogonal Decomposition (POD) method is proposed, which can analyze the spatial distribution characteristics of rainfall fields to solve data shortages. To demonstrate the feasibility and advantages of the
\end{abstract}

\footnotetext{
${ }^{*}$ Corresponding author.

E-mail address: zhangxiang@whu.edu.cn
} 
proposed methodology, four districts and counties (Hongshan District, Jianli County, Sui County, and Xuanen County) in Hubei province in China were selected as case studies. The principal results are as follows. (1) The proposed method is effective in analyzing the spatiotemporal variability of the rainfall field to reconstruct rainfall data in ungauged basins. (2) Compared with the commonly used Thiessen Polygon method, the Inverse Distance Weighting method, and the Kriging method, POD is more accurate and convenient, and the root mean squared error is reduced from $3.22,1.83,2.19$ to 2.09 ; the correlation coefficients are improved from $0.60,0.85,0.79$ to 0.89 , respectively. (3) The POD method performs particularly well in simulating the peak value and the peak time and can offer a meaningful reference for analyzing the spatial distribution of rainfall.

Keywords: Rainfall; Proper orthogonal decomposition; Spatiotemporal variations; Ungauged basin; Interpolation

\section{Introduction}

Rainfall process is a highly heterogeneous process covering an extensive range of scales in time and space (Marani, 2005; Nicótina, 2008). As the main input term of hydrological models, the variability of rainfall constitutes a significant source of uncertainty in hydrological simulations (Alexis et al., 2004), therefore, accurate and reliable estimates of the location and intensity of precipitation are crucial for water resources management and hydrological studies (Van de Beek et al., 2010).

Applying rain gauges is one way to obtain accurate rainfall data (Sohn et al., 2010; 
Van de Beek et al., 2010), however, the limitation of this method is that it only provides point measurements and therefore lacks information on the spatial variability (Van de Beek et al., 2010) unless used in a rain gauge network with sufficient density. Lebel et al. (1987) found that if rainfall measurements are based on ground measurements only, their accuracy depends on the spatial variability of the rainfall process and the density of the rain gauge network. Faurès et al. (1995) concluded that the use of a single rain gauge can lead to large uncertainties in runoff estimations for a small-scale (4.4 ha) catchment. This literature suggests that high-density rain gauge networks are needed to capture rainfall heterogeneity to accurately describe the components of the hydrologic cycle. However, equipping and maintaining high-density rain gauge networks involves high costs (Wilco et al., 2018). In most areas, the sparse and nonuniform distribution of rain gauges does not meet the requirements of hydrologic simulations (Terink et al., 2018).

With the development of remote sensing technology, satellite rainfall monitoring has become a viable option to complement rain gauge observations. Several highresolution satellite rainfall estimates are increasingly available at a quasi-global scale (Km A et al., 2021). These products have the potential to address the temporal and spatial sampling limitations of rainfall stations. However, remotely sensed rainfall products are subject to systematic bias and random error (Kidd, 2001), and their performance is highly affected by the topography and climatic conditions of the investigated regions (Romilly and Gebremichael, 2011, Worqlul et al., 2014, BaezVillanueva et al., 2018, Mekonnen et al., 2021). In addition, to represent the full spatial 
distribution of rainfall, massive satellite data is required, however, due to satellite orbit limitations, many satellite measurements are not continuous, and long records of satellite data are extremely limited (Segond et al., 2006).

The fusion of measured and satellite rainfall data is a general trend in quantitative rainfall analysis, and the consistent spatial and temporal resolution of multi-source data is a prerequisite for data fusion. In addition, different engineering projects have different demands for rainfall data with different spatial and temporal resolutions. Therefore, the interpolation method of precipitation, i.e., the spatial variation analysis of precipitation, has been widely studied (Dey and Mujumdar, 2019). There are various spatial interpolation methods for rainfall data, such as the Thiessen Polygon method, the Inverse Distance Weighting method (IDW) method, and the Kriging method, which are commonly used (Jin and Heap, 2011). The Thiessen Polygon method proceeds as follows (Croley and Hartmann, 1985): the interpolated points are connected two by two and the vertical bisectors of the connecting lines are made. The vertical bisectors intersect to form several polygons, thus dividing the large watershed into several subregions, each with a rainfall station, and the rainfall value in each region is represented by the measured value of the rainfall station within the region. The Thiessen Polygon method assumes that rainfall varies abruptly across the boundary of the polygon and that rainfall is uniformly distributed within the sub-region, which is unphysical. The IDW method assumes that the interpolated points are more influenced by the closer stations than the far ones, and thus uses the reciprocal of the squared distance as the weighting factor (Chen et al., 2015). The disadvantage of this method is that the 
interpolation error is large when the data is significantly different from the neighboring points. The Kriging method (Armstrong and Matheron, 1986) considers not only geographical features but also spatial correlation and variability of rainfall data and uses a covariance function to make the variables unbiased and optimal. However, when there are few rainfall stations, it is quite difficult to obtain the covariance function necessary for the kriging method, which will also affect the interpolation effect to some extent (Gilbert and Simpson, 1985). In addition, these methods are interpolated for a single time point, and the acquisition of rainfall processes for long time series requires iterative operations, which is very time-consuming.

To accurately and efficiently simulate the rainfall processes with high temporal resolution in large basins, it is crucial is to fully consider the heterogeneity of rainfall fields. Many factors may contribute to the variability of precipitation, including tropical disturbances, El-Niño Southern Oscillation, and Madden-Julian Oscillation, while the influence of spatial factors is the most significant (Pariyar et al., 2020). Variations in rainfall processes at different locations are often directly reflected in the temporal distribution of rainfall; therefore, they can be considered as "spatiotemporal variations". For example, owing to the different locations of sites B and A, rainfall at site B might occur later than at site $\mathrm{A}$, and the rainfall pattern at site B might be gentler (steeper) owing to weakening (enhancement) of the air mass (Palynchuk and Guo, 2011; Lin and Jhong, 2015). Several scholars have tried to represent rainfall in continuous space and time mathematically. For instance, Northrop (1998) developed a spatial-temporal model based on the Bartlett-Lewis process for the temporal evolution of storm cells and the 
Neyman-Scott process for spatial arrivals. Wilks (1998) proposed an extension from a single station to multiple stations by driving single-station models at various locations in New York with temporally independent but spatially correlated random numbers. Cowpertwait et al. (2002) proposed a spatial-temporal model based on the NeymanScott mechanism and tested the model on nine stations in Italy. Yang et al. (2005) used generalized linear models for the generation of multisite rainfall series in Southern England. Abas et al. (2014) presented a stochastic rainfall model for the generation of hourly rainfall data in an urban area in Malaysia. However, due to the influence of topography and climate, there is a large spatial gradient in rainfall intensity, such models currently assume spatial stationarity is not credible. (Alam and Elshorbagy, 2015; Kwon et al., 2016). Improvements to include nonstationary properties of precipitation, to represent (1) the temporal characteristics of rainfall, and (2) the spatial features of the data such as the location of high values of rainfall, topographic effects, or spatial continuity, are still under development (Wheater et al., 2005).

Proper Orthogonal Decomposition (POD) is an effective dimensionality reduction method that uses basis functions extracted from a large number of known data to describe the original data in an optimal approximation (Aubry, 1991). Because of its ease of interpretation and broad applicability to data from both simulations and experiments (Kutz et al., 2016), the POD technique is now used widely in fields such as fluid mechanics, aerodynamics, economics, statistics, psychology to identify and analyze spatial distribution characteristics of some variables. For example, Meric et al. (2012) applied the POD method to extract information on the dynamic spatiotemporal 
evolution of global stock markets during the 2008 economic crisis, Cheveigné and

131 Simon (2007) applied the POD method to neuroscience to extract the evolutionary

132 structure of neurons in the human brain, and Varraso et al. (2012) used POD to extract

133 and analyze the main spatial transmission of epidemic characteristics. However, this

134 method has rarely been used in rainfall analysis. The structure of a vibration field, flow

135 field, or aerodynamic field is similar to that of a rainfall field, and these fields change

136 constantly with time and space. The velocity in a flow field and the pressure in an

137 aerodynamic field are similar to the rainfall intensity in a precipitation field. Therefore,

138 the POD method also has potential for applicability in the field of rainfall simulation,

139 where it can be used to analyze the spatial distribution characteristics of rainfall fields

140 and as an interpolation-like method to obtain rainfall data in ungauged basins.

141 This study was conducted with the following two objectives: (1) to develop a

142 POD-based method to extract and analyze the spatial distribution characteristics of

143 rainfall fields. This method can be used as an interpolation-like method to reconstruct

144 rainfall processes in ungauged basins to obtain accurate simulations of rainfall

145 processes with high temporal resolution. and (2) to test the applicability of the proposed

146 method through comparative analysis with the interpolated results obtained using the

147 Thiessen Polygon method, the IDW method, and the Kriging method.

$148 \quad 2$ Methodology

149 The POD method, also known as the Karhunen-Loève procedure (Karhunen, 1946;

150 Loève, 1955), is an effective dimensionality reduction method that can extract modes

151 based on optimizing the mean square of the field variables being examined (Taira, 2017). 
It provides an objective algorithm to decompose a set of data into a minimal number of modes, each reflecting the influence of one or some physical factor on the field being decomposed, and these modes can be used to make the optimal approximate description of the original data. In this study, POD was implemented to decompose the precipitation field ( $\mathbf{X} \in \mathbf{R}^{m \times n}$ ) into several orthogonal modes, each of which may reflect the influence of one or more physical factors on the decomposed field, and the modes reflecting the influence of spatiotemporal factors can be found through comparative analysis. By reconstructing the rainfall field with the remaining modes after removing the modes reflecting spatiotemporal characteristics, the rainfall field structure independent of spatiotemporal factors can be obtained, and the rainfall processes in the ungauged basins can be reconstructed indirectly. The detailed steps and physical meanings of the variables involved in using the POD method to obtain the spatiotemporal structure of the rainfall field and to obtain data for ungauged basins are described in the following (Fig. 1).

\subsection{Sampling and pre-processing of data}

To study the influence of spatiotemporal factors on the rainfall field structure, two data sampling sessions are required. The first sample matrix $\mathbf{X}_{\mathbf{A}}$ consists of a certain rainfall process of sufficient duration at multiple stations in the study area, and the other sample matrix $\mathbf{X}_{\mathbf{B}}$ is manually spatiotemporally normalized based on the previous sample. By manual alignment (Roja et al., 2021), the rainfall processes at different stations start and end simultaneously. With the above data collation, the original determinant $\mathbf{X}_{\mathbf{A}}$ has spatiotemporal variations, and the processed determinant $\mathbf{X}_{\mathbf{B}}$ can 
174 be considered to be uniformly distributed in time and space. $\mathbf{X}_{\mathbf{A}}$ and $\mathbf{X}_{\mathbf{B}}$ can be denoted 175 as follows:

$$
\begin{aligned}
& \mathbf{X}_{\mathbf{A}}=\left[\boldsymbol{u}_{\boldsymbol{A}}\left(x, t_{1}\right), \boldsymbol{u}_{\boldsymbol{A}}\left(x, t_{2}\right), \ldots \boldsymbol{u}_{\boldsymbol{A}}\left(x, t_{n}\right)\right] \in \mathbf{R}^{m \times n} \\
& \mathbf{X}_{\mathbf{B}}=\left[\boldsymbol{u}_{\boldsymbol{B}}\left(x, t_{1}\right), \boldsymbol{u}_{\boldsymbol{B}}\left(x, t_{2}\right), \ldots \boldsymbol{u}_{\boldsymbol{B}}\left(x, t_{n}\right)\right] \in \mathbf{R}^{m \times n},
\end{aligned}
$$

178

where $n$ is the length of the rainfall duration, and $m$ reflects the number of rain gauges in the study area. In fluid dynamics, the measured state $\boldsymbol{u}\left(x, t_{n}\right)$ is related to the physics, i.e., the velocity, vorticity, and stream functions at each discretized spatial grid point. For precipitation data collected from a rainfall field, the rainfall intensity at different rain gauges at time $t_{n}$ can be denoted as $\boldsymbol{u}\left(x, t_{n}\right)$, which contains the mean of $\boldsymbol{u}(\bar{u}(x))$ and the fluctuating rainfall intensity $\boldsymbol{v}\left(x, t_{n}\right)$. It is customary to subtract $\bar{u}(x)$ before computing the POD modes. The fluctuating rainfall intensity after removing $\bar{u}(x)$ at time $n$ can be denoted as follows:

$$
\boldsymbol{v}\left(x, t_{n}\right)=\boldsymbol{u}\left(x, t_{n}\right)-\bar{u}(x)
$$

the fluctuating rainfall field after removing $\bar{u}$ at time $t_{n}$ can be denoted as follows:

$$
\begin{aligned}
& \mathbf{X}_{\mathbf{A}}=\left[\boldsymbol{v}_{\boldsymbol{A}}\left(x, t_{1}\right), \boldsymbol{v}_{\boldsymbol{A}}\left(x, t_{2}\right), \ldots \boldsymbol{v}_{\boldsymbol{A}}\left(x, t_{n}\right)\right] \in \mathbf{R}^{m \times n} \\
& \mathbf{X}_{\mathbf{B}}=\left[\boldsymbol{v}_{\boldsymbol{B}}\left(x, t_{1}\right), \boldsymbol{v}_{\boldsymbol{B}}\left(x, t_{2}\right), \ldots \boldsymbol{v}_{\boldsymbol{B}}\left(x, t_{n}\right)\right] \in \mathbf{R}^{m \times n},
\end{aligned}
$$

where $v\left(x, t_{i}\right)$ stands for the $i$-th fluctuating rainfall intensity at different stations in the study area.

\subsection{POD calculation on the sample matrices}

Various methods can be used to calculate POD, and the method selected for use in this study was the popular SVD (singular value decomposition)-based approach (Eckart and Young, 1936). The purpose of applying POD in this study is to find the mode(s) 
that reflect spatiotemporal variations of the rainfall field, and to achieve this purpose,

197 $\mathbf{X}_{\mathbf{A}}$ and $\mathbf{X}_{\mathbf{B}}$ are input to POD, respectively.

In matrix form, the data matrix $\mathbf{X}_{\mathbf{A}}$ and $\mathbf{X}_{\mathbf{B}}$ can be decomposed with SVD as follows (Fig. 2):

$$
\begin{aligned}
& X_{\mathrm{A}}=\Phi_{\mathrm{A}} \boldsymbol{\Sigma}_{\mathrm{A}} \Psi_{\mathrm{A}}{ }^{\mathrm{T}} \\
& \mathbf{X}_{\mathrm{B}}=\Phi_{\mathrm{B}} \boldsymbol{\Sigma}_{\mathrm{B}} \boldsymbol{\Psi}_{\mathrm{B}}{ }^{\mathrm{T}},
\end{aligned}
$$

where the $m \times n$ matrices $\boldsymbol{\Phi}_{\mathbf{A}}=\left[\phi_{A 1}, \boldsymbol{\phi}_{A 2}, \ldots, \boldsymbol{\phi}_{A n}\right]$ and $\boldsymbol{\Phi}_{\mathbf{B}}=\left[\phi_{B 1}, \boldsymbol{\phi}_{B 2}, \ldots, \boldsymbol{\phi}_{B n}\right]$ are composed of the modes $\phi_{A}$ and $\phi_{B} . \phi_{A}$ reflects the spatial distribution information of rainfall intensity containing rainfall spatial variability, and $\phi_{B}$ reflects the structure of the rainfall field after removing rainfall spatial variability. The $n \times n$ matrix $\boldsymbol{\Psi}_{\mathrm{A}}=\left[\boldsymbol{a}_{A 1}, \boldsymbol{a}_{A 2}, \ldots, \boldsymbol{a}_{A n}\right]$ and $\boldsymbol{\Psi}_{\mathbf{B}}=\left[\boldsymbol{a}_{B 1}, \boldsymbol{a}_{B 2}, \ldots, \boldsymbol{a}_{B n}\right]$ contain coefficients representing the temporal evolution of the modes $\boldsymbol{\Phi}_{\mathrm{A}}$ and $\boldsymbol{\Phi}_{\mathrm{B}}$, respectively. The superscript $\mathrm{T}$ represents a conjugate transpose matrix operation (Higham et al., 2017). Matrix $\Sigma$ holds singular values $\left(\sigma_{1}, \sigma_{2}, \ldots, \sigma_{n}\right)$ along its diagonal. $\lambda_{j}\left(\lambda_{j}=\sigma_{j}^{2}\right)$ is the contribution of the $j$-th mode to the total variance, indicating the influence of the $j$-th mode on the overall rainfall field. $\lambda$ is listed in descending order, i.e.: $\lambda_{1} \geq \lambda_{2} \geq \ldots \geq \lambda_{n} \geq 0$, the larger the value of $\lambda$, the greater the mode contribution. Furthermore, the percentage rainfall intensity energy contribution $(E)$ of each POD mode can be obtained as follows:

$$
E_{i}(\%)=\frac{\lambda_{i}}{\sum_{i=1}^{n} \lambda_{i}} \times 100
$$


To find the modes that characterize the spatiotemporal factors of the rainfall field,

218 the original data and the manually normalized spatiotemporal data were subjected to

219 POD in the previous section. It is assumed that the energy of the first r-order modes is

220 close to the overall energy of the precipitation field, i.e., the modes reflecting the

221 spatiotemporal influence factors of the rainfall field are included in the first r-order

222 modes, and therefore the first r-order modes are singled out for further comparison and

223 analysis.

$$
\sum_{j=1}^{r} \lambda_{j} / \sum_{j=1}^{n} \lambda_{j} \approx 1
$$

By comparing the mode values, numerical distributions, and temporal evolution coefficients of the predominant modes obtained from two POD calculations with different inputs, those modes with significant differences are excluded because they are considered to be related to spatiotemporal properties. Then, a homogeneous precipitation field is reconstructed with the remaining predominant modes. The fluctuating rainfall intensity $v(x, t)$ can be expressed as a linear superposition of all orthogonal modes $(\phi(x))$ multiplied by their corresponding time factors $(\boldsymbol{a}(t))$ :

$$
\boldsymbol{v}(x, t)=\sum_{j=1}^{\mathrm{r}} \boldsymbol{a}_{j}(t) \boldsymbol{\phi}_{j}(x)
$$

where $j$ is the subscript of the remaining modes after removing those modes related to spatiotemporal properties. Then, the precipitation field can be reconstructed as follows:

$$
\mathbf{X}_{P O D}(x, t)=\bar{u}(x)+\sum_{\mathrm{j}=1}^{r} \boldsymbol{a}_{j}(t) \phi_{j}(x)
$$

Because the temporal and spatial variations of the precipitation field are eliminated, 
precipitation processes in the ungauged basin can be represented by precipitation

239 processes at an adjacent station (Fig. 3).

\subsection{Verification methods}

To assess the performance of the methods used in this study, we used three performance statistics widely used to compare the model performance such as Root Mean Square Error (RMSE), Relative Error (RE), and Correlation correlation (Corr).

Based on the results, performance of these methods is presented in detail.

\section{(1) Root mean squared error (RMSE)}

The RMSE (Eq. (9)) estimates the standard deviation between the observed value and the simulated value. A small RMSE value indicates better performance whereas a higher value indicates a poor performance.

where $Q_{O}$ and $Q_{m}$ refer to the observed and simulated values, respectively, and $\mathrm{T}$ is the total number of observations.

\section{(2) Relative error (RE)}

The RE represents the magnitude of the deviation of the simulated value from the 254 true value and is calculated as follows:

$$
R E=\frac{1}{T}\left[\sum_{t=1}^{T}\left|1-\frac{Q_{m}^{\mathrm{t}}}{Q_{o}^{\mathrm{t}}}\right|\right] \times 100 \%
$$

\section{(3) Correlation coefficient (Corr)}

The Corr is a statistical indicator to reflect the closeness of the correlation between

258 variables and its value ranges between -1 and 1 representing a perfect negative and 
positive correlation, respectively. The correlation coefficient is calculated by the product-difference method and is based on the deviation of the two variables from their respective means. The formula for calculating correlation can be represented as:

$$
\operatorname{Corr}(X, Y)=\frac{\operatorname{Cov}(X, Y)}{\sqrt{\operatorname{Var}[X] \operatorname{Var}[Y]}}
$$

where $\operatorname{Cov}(X, Y)$ is the covariance of $X$ and $Y, \operatorname{Var}[X]$ is the variance of $X$, and

$\operatorname{Var}[Y]$ is the variance of $Y$

\section{Study area and data}

Hubei Province is located in central China, between latitude $29^{\circ} 01^{\prime} 53^{\prime \prime}-33^{\circ} 6^{\prime} 47^{\prime \prime}$

$\mathrm{N}$ and longitude $108^{\circ} 21^{\prime} 42^{\prime \prime}-116^{\circ} 07^{\prime} 50^{\prime \prime} \mathrm{E}$ with a total area of $185900 \mathrm{~km}^{2}$. Mountains, hills, and plain lakes account for $56 \%, 24 \%$, and $20 \%$ of the total area of Hubei province, respectively. Except for the alpine climate in the high mountain areas, most parts of the province belong to the subtropical monsoon humid climate, with sufficient light energy, abundant rainfall, and long frost-free periods. The average annual precipitation in Hubei province is between 800 and $1600 \mathrm{~mm}$. The regional distribution of precipitation shows a decreasing trend from south to north (Fig. 4), with a maximum of 1400-1600 mm in the southwest and a minimum of $800-1000 \mathrm{~mm}$ in the northwest. The seasonality of precipitation distribution is obvious, with the most in summer $(300-700 \mathrm{~mm})$ and the least in winter (30-190 mm), respectively. The rainy season is from mid-June to midJuly, with the largest rainfall and intensity. To study the applicability of the method recommended in this paper, we selected several extreme rainstorms in Hubei Province

279 for analysis. Hourly rainfall data at 56 stations in Hubei province are downloaded from 


\section{Results}

282

283

284

285

286

287

288

289

290

291

292

293

294

295

296

297

298

299

300

301

302

\subsection{Reconstruction of rainfall processes at ungauged basins}

Because of the large spatially varying gradients of precipitation in Hongshan District, Jianli County, Sui County, and Xuanen County in Hubei province, these locations are assumed to be the ungauged basins for rainfall reconstruction (Table 1). The multiple extreme rainfall data of Hubei Province in 2020 were used as the input of POD to obtain the modes with spatiotemporal variability, and the data processed by manual spatiotemporal normalization were input to obtain the modes with spatiotemporal variability removed. Fig. 5 shows the energy contribution and the cumulative energy contribution of the modes decomposed by POD with raw data and processed data as input, respectively. The energy contributions of the modes obtained from the POD of raw data show a larger descending trend, where the cumulative energy contribution ratio of the first two modes is approximately $10 \%$ higher than that of the modes obtained by processed data, which indicates that these modes are more representative. Starting from the fifth mode, the energy contribution of processed data becomes larger than that of the raw data. And after the ninth mode, the cumulative energy contribution rates of the two compared cases are almost the same. In both cases, the cumulative energy contribution rates of the first six modes exceed $80 \%$ and thus these modes are selected as the predominant modes for subsequent analysis and reconstruction of the precipitation field.

Fig. 6 shows the modes and the corresponding time factors before spatiotemporal normalization, i.e., raw data is used as input to the POD. As the energy contribution of 
the mode decreases, a higher proportion of null values is observed for modes with values close to zero, which is consistent with the physical meaning of the energy contribution. In addition, as the energy contribution rate decreases, the time factor corresponding to the mode fluctuates more frequently, which indicates that the mode becomes more unstable as the energy contribution rate decreases. in a certain physical sense, and the corresponding time factor reflects the trend of the mode over time. Because the energy contribution of the first mode exceeds $27.44 \%$, it can be assumed that the reconstruction based on the first mode reflects the main structure of the precipitation field. The values of the first mode are mostly negative and the corresponding time factor is in the inverted triangular shape; therefore, a positive triangular rainfall intensity process can be obtained by multiplying the first mode and its corresponding time factor. The major mode values of the second mode are positive, with the corresponding time factor appearing in the shape of a triangle. A similar rainfall process that increases firstly and then decreases can be obtained by multiplying the two. This can be interpreted as the structure of this precipitation field is a single-peak rainfall process. The values of the third to sixth modes are mostly around zero, and their corresponding time factors fluctuate frequently. This indicates that they play little role in the final results of the reconstructed rainfall pattern, but there may still be modes reflecting spatiotemporal factors in them.

To find the modes characterizing the variations of time and space, the processed data are used as input to POD to get the modes after spatiotemporal normalization. 
Similar to the modes obtained from the original data as input, the proportion of null values and the fluctuation frequency of the time factors increase with the decrease of the energy contribution of the modes. However, the predominant modes and their corresponding time factors change slightly due to different inputs (Fig. 7). Compared with the first mode before spatiotemporal normalization, the values of the first mode after spatiotemporal normalization are also mostly negative, decreasing from northwest to northeast and slightly higher in the southeast. The main pattern of precipitation after spatiotemporal normalization is also a single-peaked rainfall with a backward peak, which indicates that the main pattern of precipitation does not change significantly before and after the normalization of time and space. The distribution of the values of the fourth mode is almost the same before and after normalization. The values of the northeast, and the trends of time factors of the fifth modes before and after the modes do not reflect the influence of spatiotemporal factors on the structure of the precipitation field. However, the second, third, and sixth modes change significantly due to the normalization of time and space. Therefore, it can be assumed that the superimposed effect of these three modes reflects the spatiotemporal variations of the precipitation field. Therefore, the reconstructed precipitation field after removing these three modes can be regarded as the result of removing the spatiotemporal variations.

Fig. 8 and Fig. 9 show the reconstructions of precipitation fields before and after spatiotemporal normalization (removing the second, third, and sixth modes), 
respectively. The comparison shows that the rainfall processes at different stations are more consistent and the peaks are more pronounced after the removal of spatiotemporal variations.

\subsection{Comparative analysis of different methods}

Based on the results obtained in the previous section, the rainfall processes at the ungauged sites are reconstructed according to the method introduced in section 2.3. To illustrate the implementability and superiority of the proposed method, three most commonly used interpolation methods in current hydrological engineering: the Thiessen Polygon method, the IDW method, and the Kriging method, were selected for comparative study. The rainfall data obtained by different methods as well as the measured rainfall data in the ungauged stations are shown in Fig. 10. It can be seen that a 24-h rainfall varies in magnitude and time distribution in different areas of Hubei Province, and the interpolation results of different methods applied in different areas also have great differences. The Thiessen Polygon method is rough from the mechanism, and the interpolation of rainfall peak values and peak times is not accurate enough, especially in Jianli County where the rainfall gauge distribution is relatively sparse. The interpolation results of peak present time in both Hongshan District and Sui County are method has a small simulation of the early and late rainfall processes but compared with the other three methods, it has a more accurate simulation of the peak value and peak 
time. The median and arithmetic mean of the relative errors of the POD simulations for the peak portion of the rainfall are the smallest, which is very meaningful for hydrological design (Fig. 11).

Fig. 12 and Table 2 show the Corrs between the interpolated results and the measured values, as well as the RMSEs. It can be found that the results obtained by the Thiessen Polygon method have a large error, and the accuracy of the interpolation results for rain intensity above $5 \mathrm{~mm} / \mathrm{h}$ is low, with a Corr of only 0.6 and an RMSE as high as $3.22 \mathrm{~mm} / \mathrm{h}$. The interpolation results of Kriging method are mostly a little smaller than the measured values, which is unfavorable from the perspective of practical engineering safety. The Corr of the Kriging method is 0.79 and the RMSE is $2.19 \mathrm{~mm} / \mathrm{h}$. The interpolation results of the IDW method are more accurate, with an RMSE of only $1.83 \mathrm{~mm} / \mathrm{h}$ and a Corr of 0.85 , but the interpolation of the IDW method for rainfall exceeding $15 \mathrm{~m} / \mathrm{h}$ tends to be small. Perhaps because the extracted modes contain not only the spatial and temporal characteristics of the rainfall field but also a part of the initial and final rainfall, the POD method in this case is inaccurate in simulating rainfall amounts less than $5 \mathrm{~mm} / \mathrm{h}$ at the beginning and end of the rainfall period, resulting in an RMSE of $2.09 \mathrm{~mm} / \mathrm{h}$. However, the POD method is more accurate for rainfall over $10 \mathrm{~mm} / \mathrm{h}$ and the Corr exceeds 0.89 .

\section{Discussion and Conclusions}

Spatial interpolation of rainfall has been regarded as the most common method to obtain rainfall data in ungauged basins, and the non-consistency of rainfall spatial distribution is the source of error in the interpolation method. In this study, POD is 
introduced to analyze the spatiotemporal variations of rainfall fields and is used as an interpolation-like method to obtain rainfall data in ungauged basins.

POD is a dimensionality reduction method that can be used to analyze the spatial distribution characteristics of different variable fields. Similar to its application to extract the flow structure in fluid mechanics (Redha et al., 2018), information on dynamic market transactions in finance (Meric et al., 2012), neuronal evolutionary structures in neuroscience (Cheveigné and Simon, 2007), and virus transmission characteristics in epidemiology (Varraso et al., 2012), our study demonstrated that POD can efficiently identify and extract the structures of the rainfall field. By comparing the results obtained from two different sets of data as input, it is possible to find the modes that reflect the characteristics of the spatial distribution of the rainfall field, and the rainfall field reconstructed by removing these modes can be regarded as spatially uniformly distributed. Based on this, the rainfall processes in the ungauged stations can be reconstructed using the measured rainfall data from adjacent stations. This is particularly advantageous for urban areas with large spatially varying rainfall gradients. In the application to the case study area in Hubei Province, POD was proven an effective and reliable approach for obtaining rainfall data in ungauged basins. The first advantage of the proposed method is that compared with the traditional method, the proposed method has a significant improvement in the simulation of the peak value and peak time. Second, the use of the POD method to remove spatiotemporal correlation modes to reconstruct rainfall in ungauged basins is once and for all, and it is more efficient to obtain long series of rainfall data with the POD method than other methods 
that require repeated interpolations. However, despite the above advantages, this method has the potential for further improvement. For example, how to separate the parts of the same mode that may contain different characteristics of the rainfall field and how to quantitatively identify the modes that characterize spatial and temporal properties remain to be discussed. It is hoped that this study will serve as a stepping stone for the readers to become familiar with POD, analyze complex issues associated with spatial heterogeneity of rainfall fields, and further advance the developments of this method in analyzing the effects of other factors (e.g., distance between stations and the ocean, slope, slope direction, wind speed) on the spatial distribution of rainfall data.

\section{Data Availability Statement}

All data included in this study are available upon request by contact with the corresponding author.

\section{Acknowledgments}

This research is supposed by the National Key Research and Development Program of China (No.2019YFC0408901) and the National Natural Science Foundation of China (No.41890823). We express our gratitude to the reviewers and editors for their comments on the manuscript. 


\section{References}

432 1. Abas, N. et al. A comparative study of mixed exponential and Weibull distributions 433 in a stochastic model replicating a tropical rainfall process. Theoretical and Applied 434 Climatology. 118(3), 597-607 (2014).

2. Alam, M. S., and Elshorbagy, A. Quantification of the climate change-induced variations in intensity-duration-frequency curves in the Canadian prairies. Journal of Hydrology. 527, 990-1005 (2015).

3. Alexis, Berne, and Guy, Delrieu. Temporal and spatial resolution of rainfall measurements required for urban hydrology. Journal of Hydrology. 299(3-4), 166-179 (2004).

4. Armstrong, M., and Matheron, G. Disjunctive kriging revisited. I, II. Math Geol. (8), 711-728,729-742 (1986).

5. Aubry, N. On the hidden beauty of the proper orthogonal decomposition.

Theoretical and Computational Fluid Dynamics. 2(5), 339-352 (1991).

6. Baez-Villanueva, O. M., Zambrano-Bigiarini, M., Ribbe, L., Nauditt, A., Giraldo-

Osorio, J. D., and Thinh, N. X. Temporal and spatial evaluation of satellite rainfall estimates over different regions in Latin-America. Atmospheric Research. 213(NOV.), 34-50 (2018).

7. Chen, C., Zhao, N., Yue, T., and Guo, J. A generalization of inverse distance weighting method via kernel regression and its application to surface modeling. Arabian Journal of Geosciences. 8, 6623-6633 (2015).

8. Cheveigné AD, and Simon, J. Z. Denoising based on time-shift PCA. Journal of 
Neuroscience Methods. 165(2), 297-305 (2007).

9. Cowpertwait, P., Kilsby, C. G., and O'Connell, P. E. A space-time neyman-scott model of rainfall: empirical analysis of extremes. Water Resources Research. 38(8), 61-6-14 (2002).

10. Croley, T. E., and Hartmann, H. C. Resolving thiessen polygons. Journal of Hydrology. 76(3-4), 363-379 (1985).

11. Dey, P., and Mujumdar, P. P. On the uniformity of rainfall distribution over India. Journal of Hydrology. 578, 124017 (2019).

12. Eckart, C., and Young, G. The approximation of one matrix by another of lower rank. Psychometrika. 1(3), 211-218 (1936).

13. Higham, J. E. et al. Using modal decompositions to explain the sudden expansion of the mixing layer in the wake of a groyne in a shallow flow. Advances in Water Resources. 107: 451-459 (2017).

14. Gilbert, R. O., and Simpson, J. C. Kriging for estimating spatial pattern of contaminants: potential and problems. Environmental Monitoring and Assessment. 5(2), $113(1985)$.

15. Jin, L., and Heap, A. D. A review of comparative studies of spatial interpolation methods in environmental sciences: performance and impact factors. Ecological Informatics. 6(3-4), 228-241 (2011).

16. JM Faurès, Goodrich, D. C., Woolhiser, D. A., and Sorooshian, S. Impact of smallscale spatial rainfall variability on runoff modeling. Journal of Hydrology. 173(1), 309$326(1995)$. 
17. Karhunen, K. Zur spektral theorie stochasticher prozesse. Annales Academiae Scientiarum Fennicae. 34, 13 (1946).

18. Kidd, C. Satellite rainfall climatology: a review. International Journal of Climatology. 21(9), 1041-1066 (2010).

19. Km, A., Amm, B., and Taw, A. Effect of temporal sampling mismatches between satellite rainfall estimates and rain gauge observations on modelling extreme rainfall in the upper awash basin, Ethiopia. Journal of Hydrology. 598(7), 126467 (2021).

20. Kutz, J. N., Brunton, S. L., Brunton, B. W., and Proctor, J. L. Dynamic Mode Decomposition: Data-Driven Modeling of Complex Systems. Society for Industrial and Applied Mathematics Philadelphia (2016).

21. Kwon, Hyun-Han, Lima, Carlos, H. R., Kim, and Jin-Young. A bayesian beta distribution model for estimating rainfall IDF curves in a changing climate. Journal of Hydrology. 540, 744-756 (2016).

22. L Nicótina, Celegon, E. A., Rinaldo, A., and Marani, M. On the impact of rainfall patterns on the hydrologic response. Water Resources Research. 44(12), 15-28 (2008).

23. Lebel, T., Bastin, G., Obled, C., and Creutin, J. D. On the accuracy of areal rainfall estimation: a case study. Water Resources Research, 23(11), 2123-2134 (1987).

24. Lin, G. F., and Jhong, B. C. A real-time forecasting model for the spatial distribution of typhoon rainfall-sciencedirect. Journal of Hydrology. 521, 302-313 (2015).

25. Loève, M., Probability Theory, Princeton, N.J.: D Van Nostrand, (1955).

26. Marani, M. Correction to "non-power-law-scale properties of rainfall in space and 
time”. Water Resources Research. 41(8), 323-333 (2005).

27. Mekonnen, K., Melesse, A. M., and Woldesenbet, T. A. Spatial evaluation of satellite-retrieved extreme rainfall rates in the upper awash river basin, Ethiopia. Atmospheric Research. 249, 105297 (2020).

28. Meric, G., Lentz, C., Smeltz, W., and Meric, I. International evidence on market linkages after the 2008 stock market crash. International Journal of Business and Finance Research. 6(4): 45-57 (2012).

29. Northrop, P. A clustered spatial-temporal model of rainfall. Proceedings of the Royal Society A: Mathematical, Physical and Engineering Sciences. 454(1975), 1875$1888(1998)$.

30. Palynchuk, B. A., and Guo, Y. A probabilistic description of rain storms incorporating peak intensities. Journal of Hydrology. 409(1-2), 71-80 (2011).

31. Pariyar, S. K., Keenlyside, N., Sorteberg, A., Spengler, T., and Ogawa, F. Factors affecting extreme rainfall events in the south pacific. Weather and Climate Extremes. 29(6), $100262(2020)$.

32. Redha, Wahidi, Semih, M., Olcmen, and James, et al. Different approaches to applying pod analysis to $3 \mathrm{~d} 3 \mathrm{c}$ data in a large measurement volume. Fluid mechanics research. 45(3), 187-201 (2018).

33. Roja, C., Hamza, V., Milind M., Ingle, S. T., Kuttippurath, J. Changes in large-scale circulation over the indo-pacific region and its association with 2018 kerala extreme rainfall event. Atmospheric Research. 263, 105809 (2021).

34. Romilly, T. G., and Gebremichael, M. Evaluation of satellite rainfall estimates over 
Ethiopian river basins. Hydrology and Earth System Sciences. 15(5), 1505-1514 (2010).

35. Segond, M. L., Onof, C., and Wheater, H. S. Spatial-temporal disaggregation of daily rainfall from a generalized linear model. Journal of Hydrology. 331(3-4), 674-689 (2006).

36. Sohn, B. J., Han, H. J., and Seo, E. K. Validation of satellite-based high-resolution rainfall products over the Korean peninsula using data from a dense rain gauge network. Journal of Applied Meteorology and Climatology. 49(4), 367-70 (2010).

37. Taira, K., Brunton, S. L., Dawson, S., Rowley, C. W., Colonius, T., and Mckeon, B. J. Modal analysis of fluid flows: an overview. AIAA Journal. 55(12), 4013-4041 (2017). 38. Terink, W., Leijnse, H., van den Eertwegh, Gé, and Uijlenhoet, R. Spatial resolutions in areal rainfall estimation and their impact on hydrological simulations of a lowland catchment. Journal of Hydrology. 563, 319-335 (2018).

39. Van, d. B. C. Z., Leijnse, H., Torfs, P., and Uijlenhoet, R. Climatology of daily rainfall semi-variance in the netherlands. Hydrology and Earth System Sciences. 15(1), 171-183 (2010).

40. Varraso, R., Garcia-Aymerich, J., Monier, F., Moual, N. L., Batlle, J. D., and Miranda, G., et al. Assessment of dietary patterns in nutritional epidemiology: principal component analysis compared with confirmatory factor analysis1,2,3. The American Journal of Clinical Nutrition. 5: 1079-1092 (2012).

41. Wheater, H. S., Chandler, R. E., Onof, C. J., Isham, V. S., Bellone, E., and Yang, C. Spatial-temporal rainfall modeling for flood risk estimation. Stochastic Environmental Research and Risk Assessment. 19(6), 403-416 (2005). 
541 42. Wilks, D. S. Multisite generalization of a daily stochastic precipitation generation 542 model. Journal of Hydrology. 210(1), 178-191 (1998).

543 43. Worqlul, A., W., Steenhuis, T., and S., et al. Comparison of rainfall estimations by 544 trmm 3b42, mpeg and cfsr with ground-observed data for the lake tana basin in Ethiopia. 545 Hydrology and Earth System Sciences. 18(12), 4871-4881 (2014).

546 44. Yang, C., Chandler, R. E., Isham, V. S., and Wheater, H. S. Spatial-temporal rainfall 547 simulation using generalized linear models. Water resources research. 41(11), $548 \quad$ p.W11415.1-W11415.13 (2005).

549 


\section{List of Tables}

551 Table 1. Latitude and longitude of the case studies

\begin{tabular}{cccc}
\hline Ungauged basins & Latitude and longitude & Refactoring templates & Latitude and longitude \\
\hline Hongshan & $114.4^{\circ} \mathrm{E}, 30.5^{\circ} \mathrm{N}$ & Xinzhou & $115.0^{\circ} \mathrm{E}, 31.0^{\circ} \mathrm{N}$ \\
Jianli & $113.1^{\circ} \mathrm{E}, 30.0^{\circ} \mathrm{N}$ & Tianmen & $113.1^{\circ} \mathrm{E}, 30.5^{\circ} \mathrm{N}$ \\
Sui & $113.8^{\circ} \mathrm{E}, 31.5^{\circ} \mathrm{N}$ & Guangshui & $113.8^{\circ} \mathrm{E}, 32.0^{\circ} \mathrm{N}$ \\
Xuanen & $109.4^{\circ} \mathrm{E}, 30.0^{\circ} \mathrm{N}$ & Hefeng & $110.0^{\circ} \mathrm{E}, 30.0^{\circ} \mathrm{N}$ \\
\hline
\end{tabular}

552

553 Table 2. Statistical indicators for validation of interpolation results of different

554 methods

\begin{tabular}{cccc}
\hline Methods & RMSE (mm/h) & Corr & RE of rain peak (\%) \\
\hline Thiessen Polygon & 3.22 & 0.60 & 83.56 \\
IDW & 1.83 & 0.85 & 47.57 \\
Kriging & 2.19 & 0.79 & 49.84 \\
POD & 2.09 & 0.89 & 32.40 \\
\hline
\end{tabular}

555 


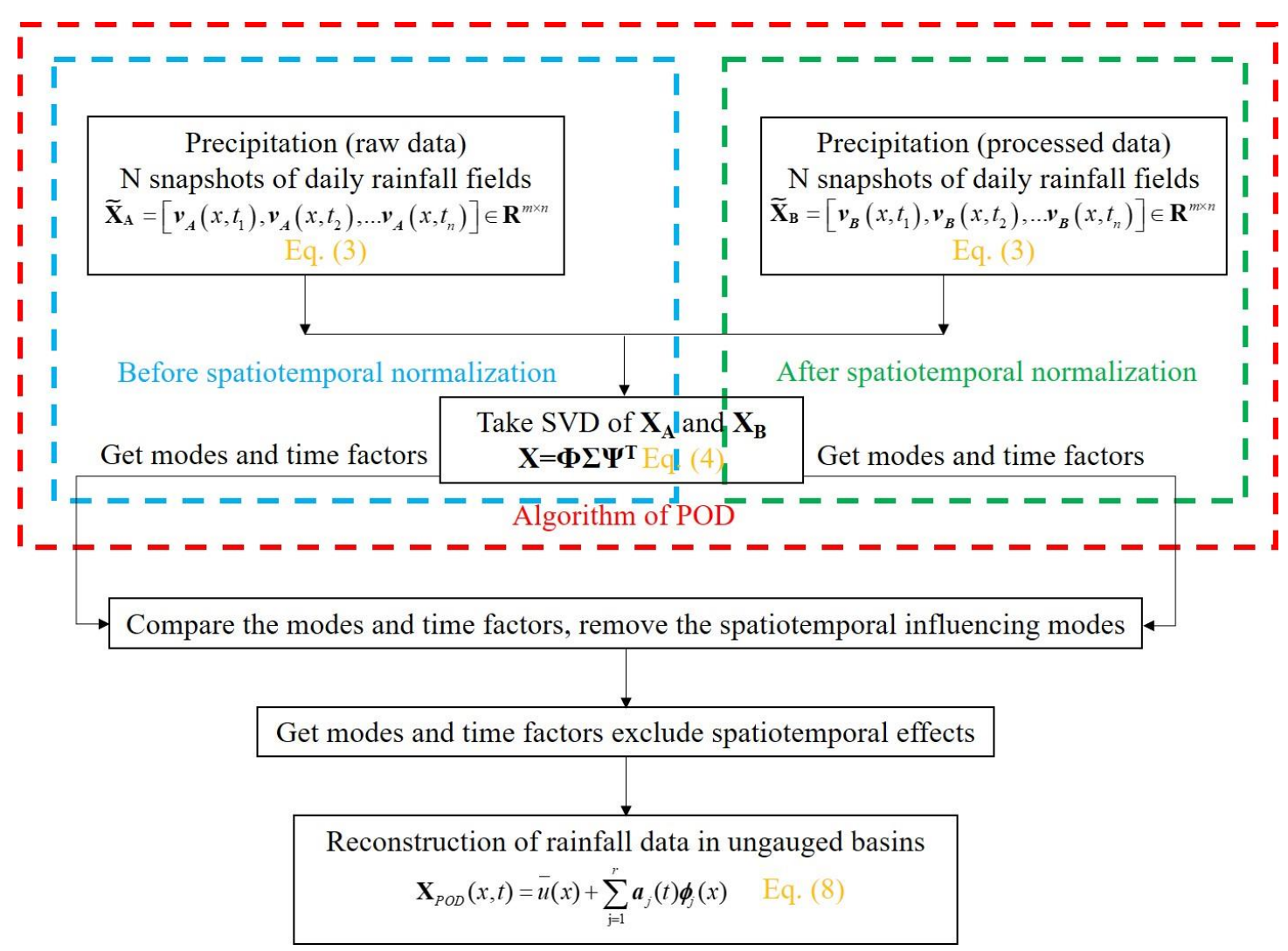

Fig. 1. Flow chart depicting the methodology proposed in this study.

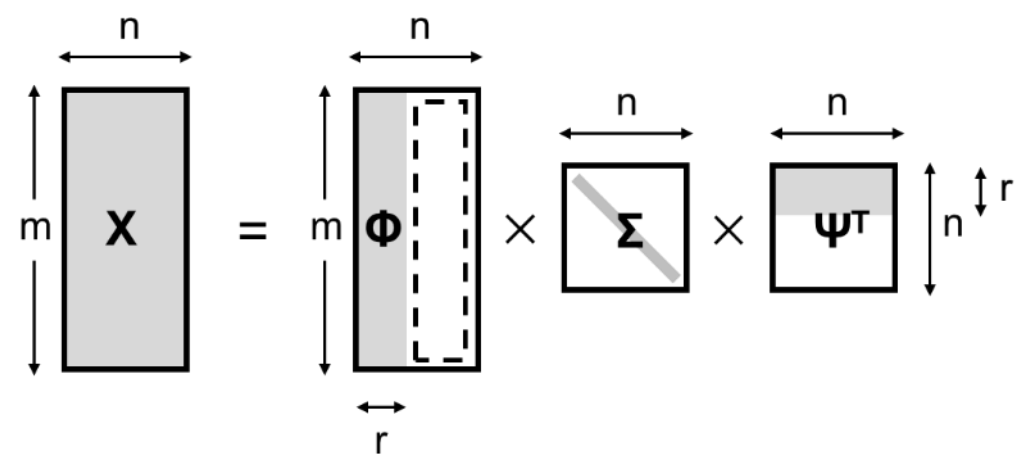

561 Fig. 2. Graphical representation of singular value decomposition. 


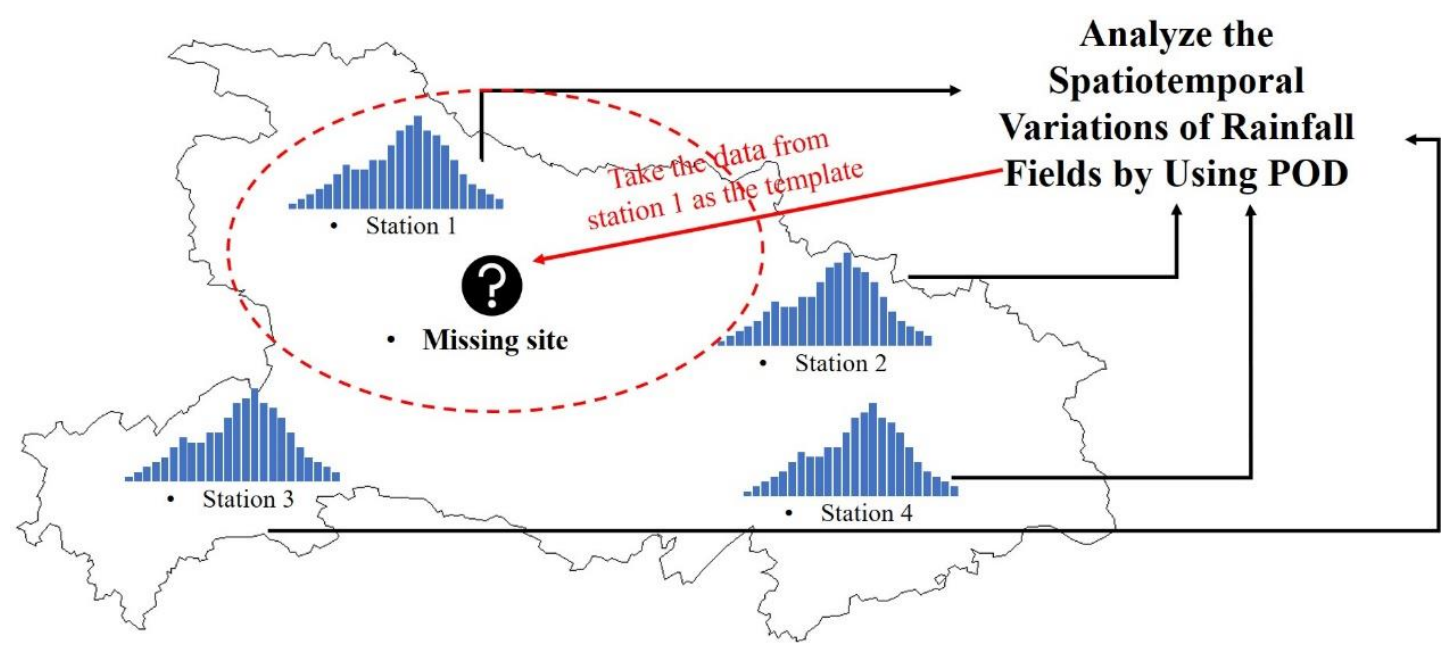

563 Fig. 3. Reconstruction of rainfall processes at ungauged basins.

564

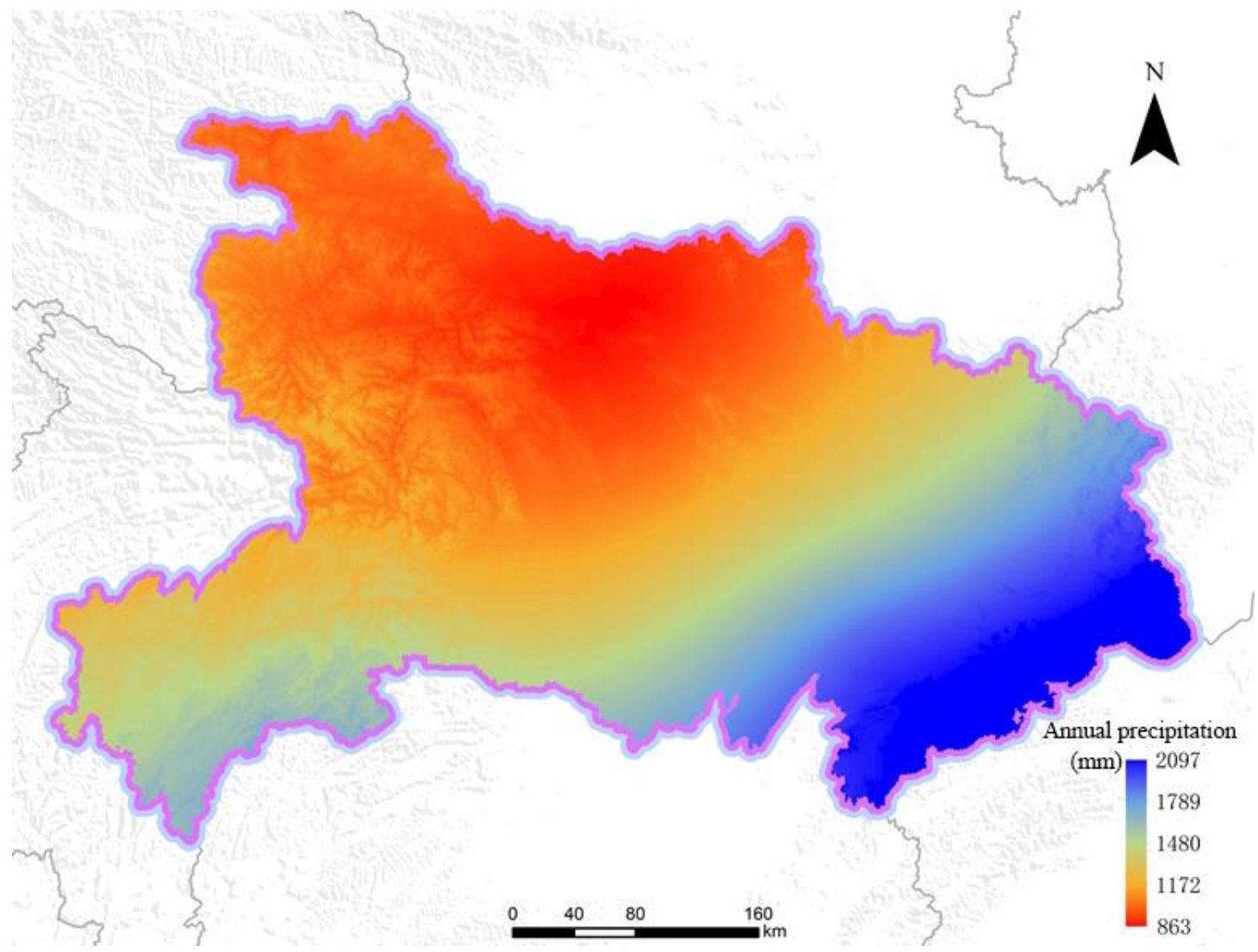

Fig. 4. Distribution of annual rainfall contours in Hubei Province. 


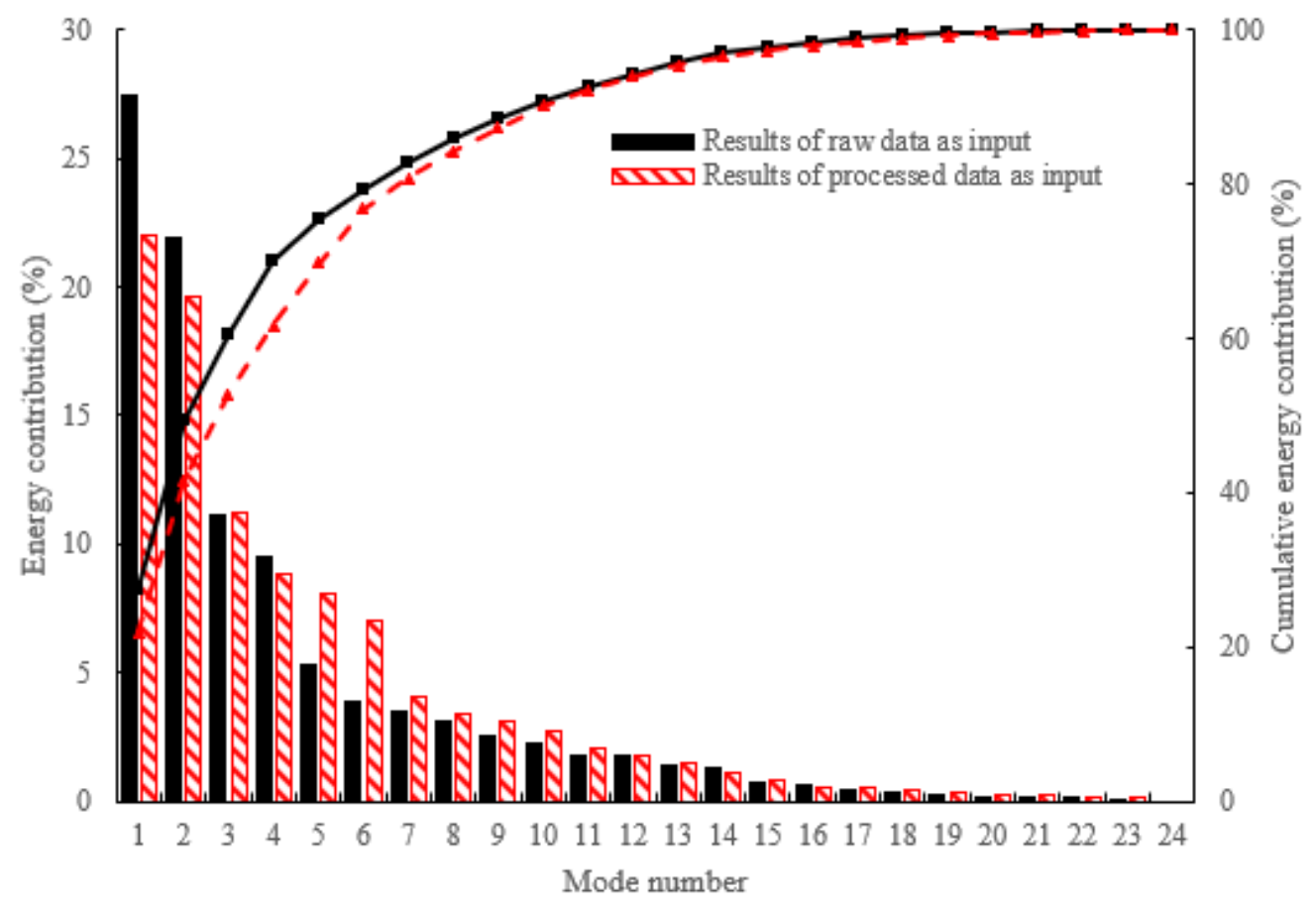

Fig. 5. The energy contribution of the modes.

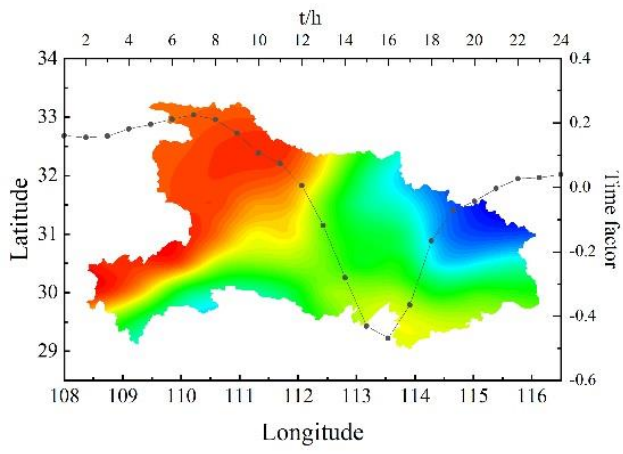

(a)The first mode

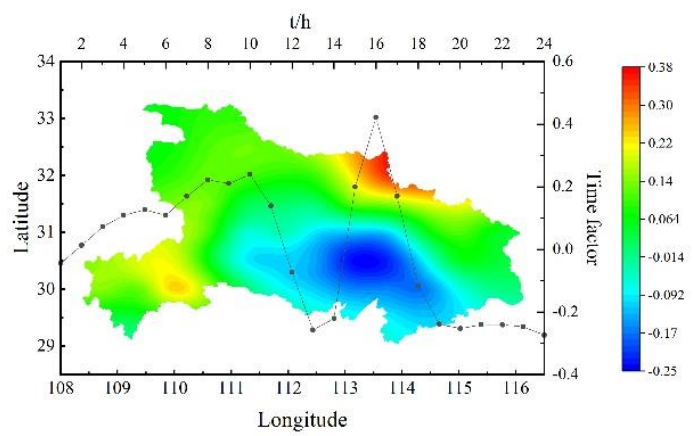

572

573
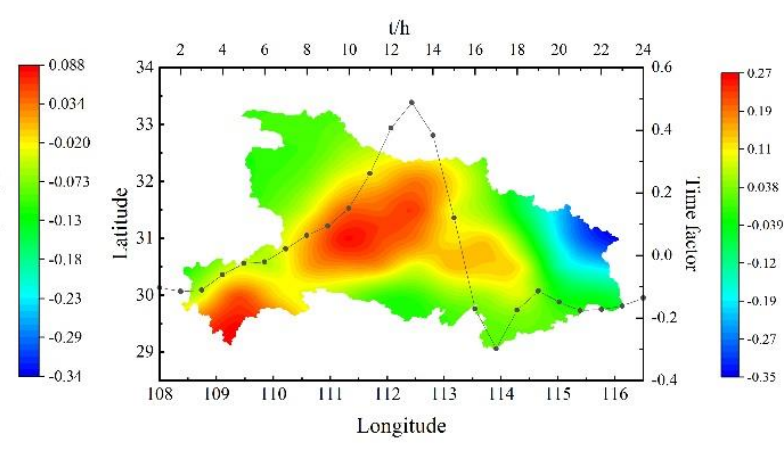

(b)The second mode

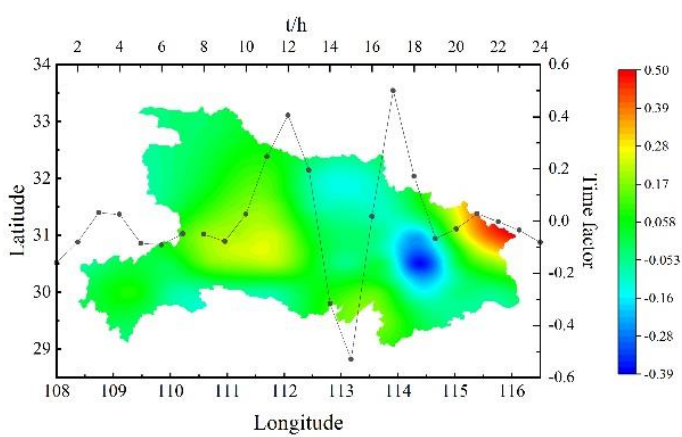

(d)The fourth mode 


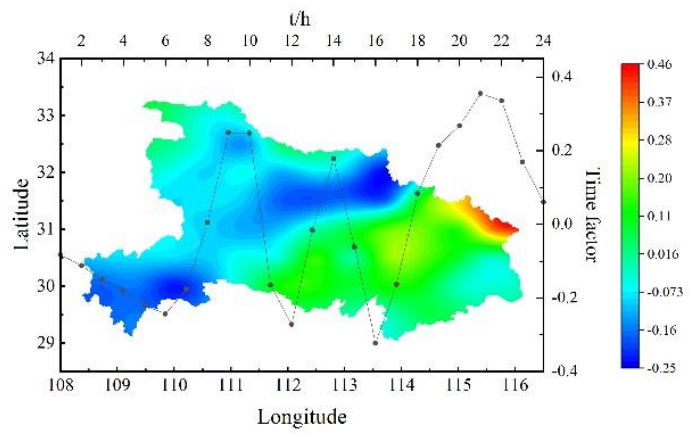

(e)The fifth mode

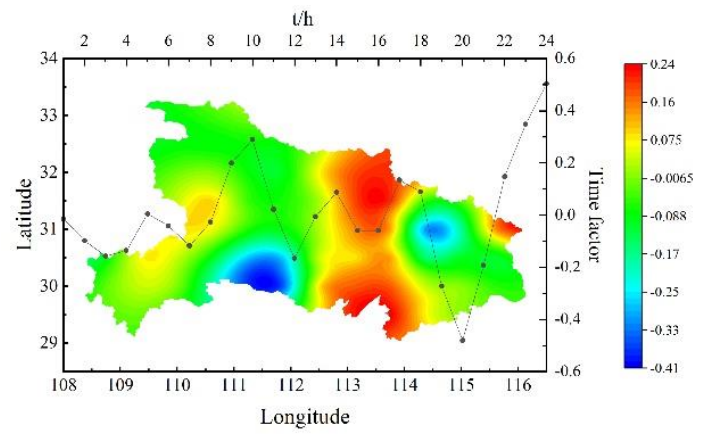

(f)The sixth mode

576 Fig. 6. Top 6 modes and corresponding time factors before spatiotemporal

577 normalization.

578

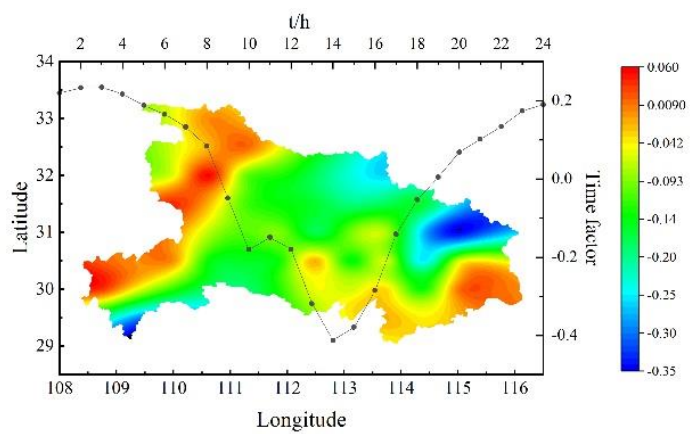

(a)The first mode

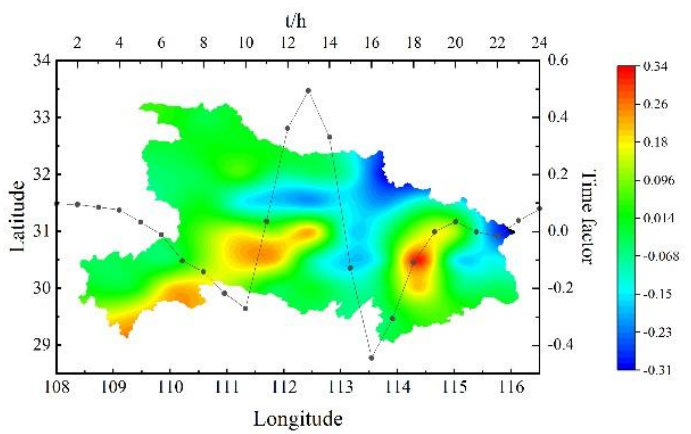

(c)The third mode

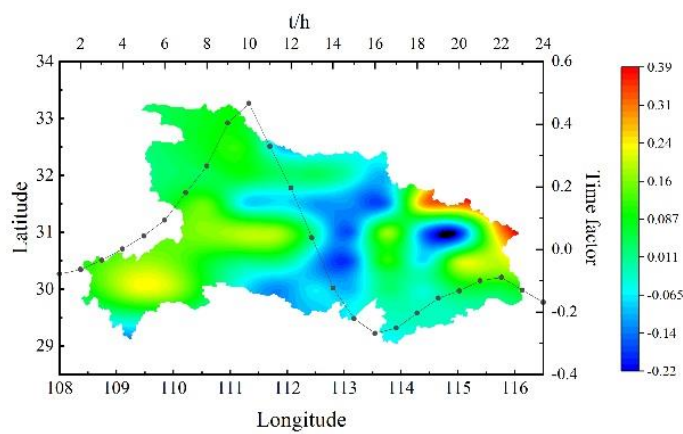

(b)The second mode

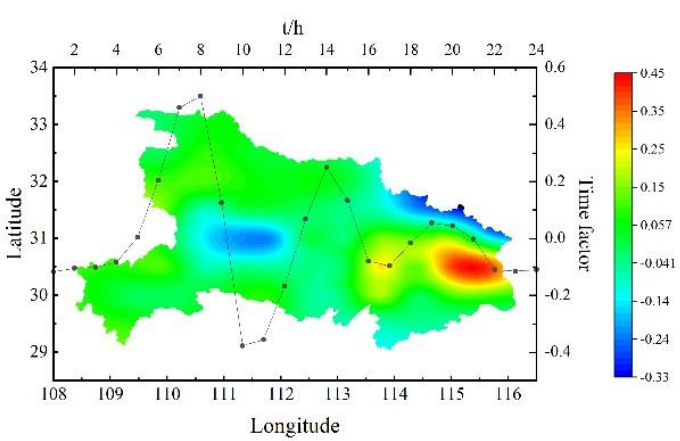

(d)The fourth mode 
583

584

585

586

587

588

589

590

591

592

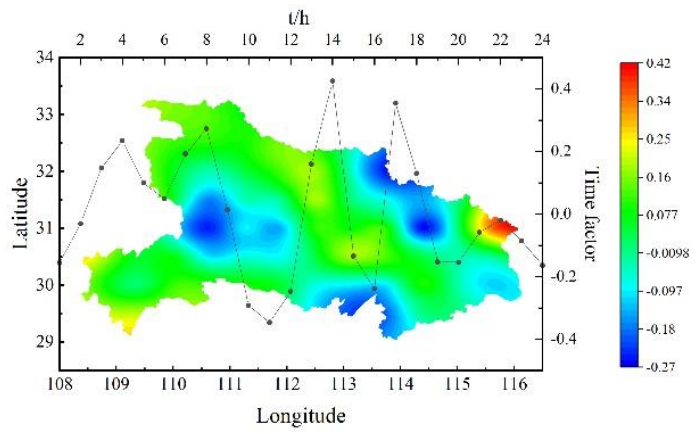

(e)The fifth mode

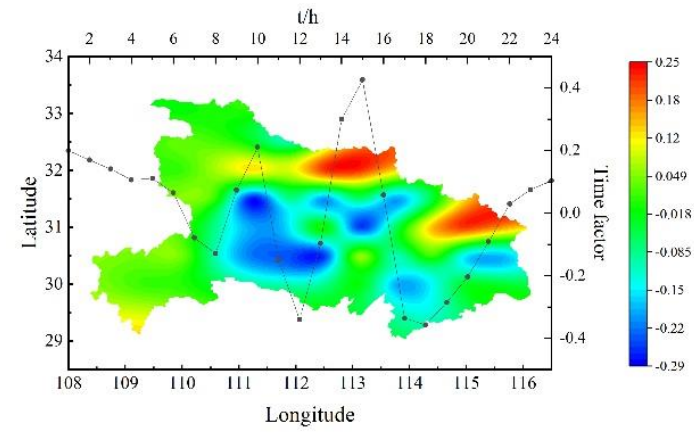

(f)The sixth mode

Fig. 7. Top 6 modes and corresponding time factors after spatiotemporal normalization.

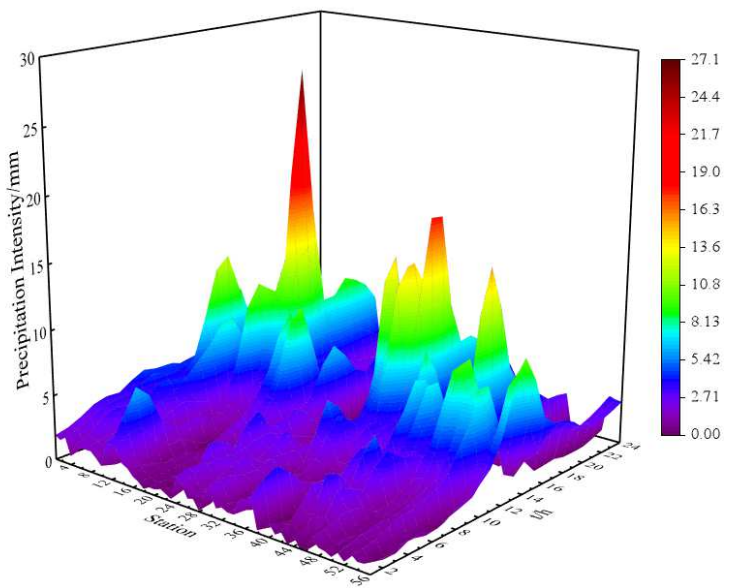

Fig. 8. The reconstructed precipitation field before spatiotemporal normalization.

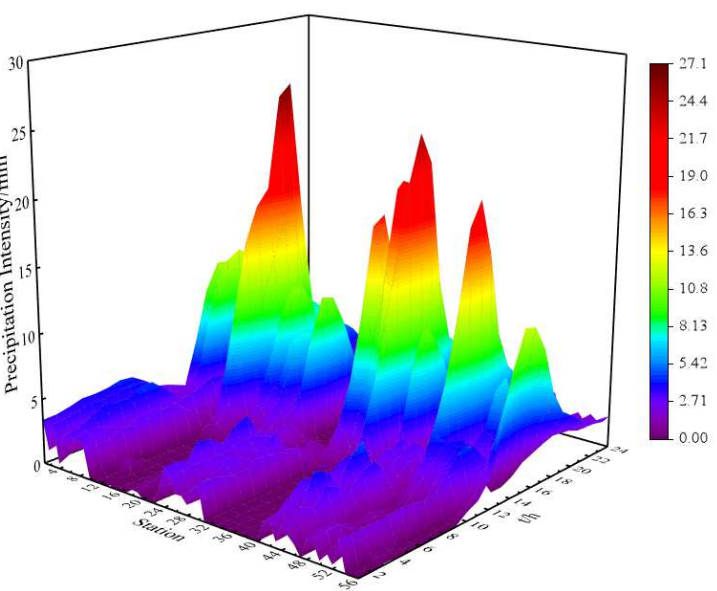

Fig. 9. The reconstructed precipitation field after removing spatiotemporal variations. 

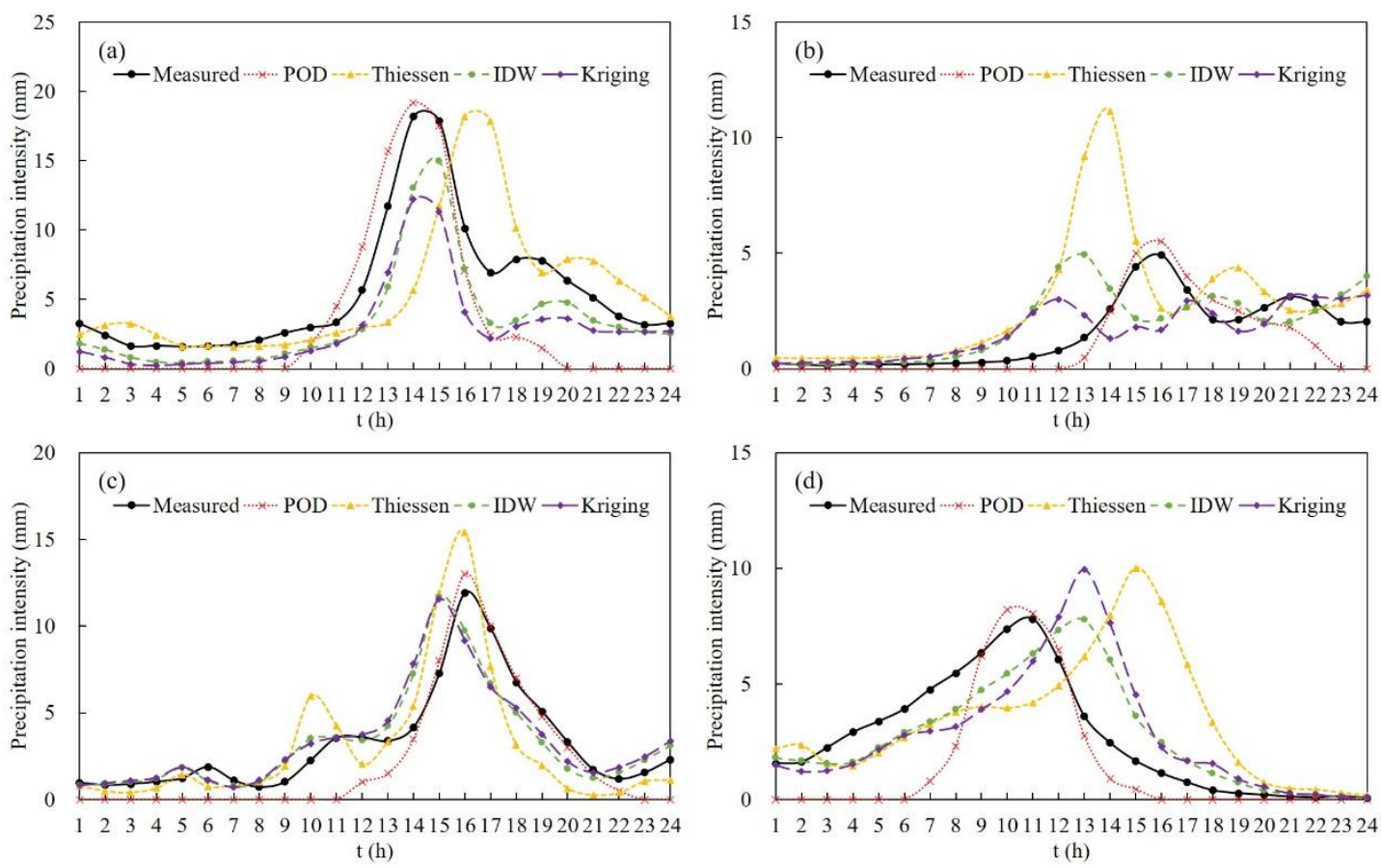

Fig. 10. A 24-h rainfall process obtained by different methods in ungauged basins: (a)

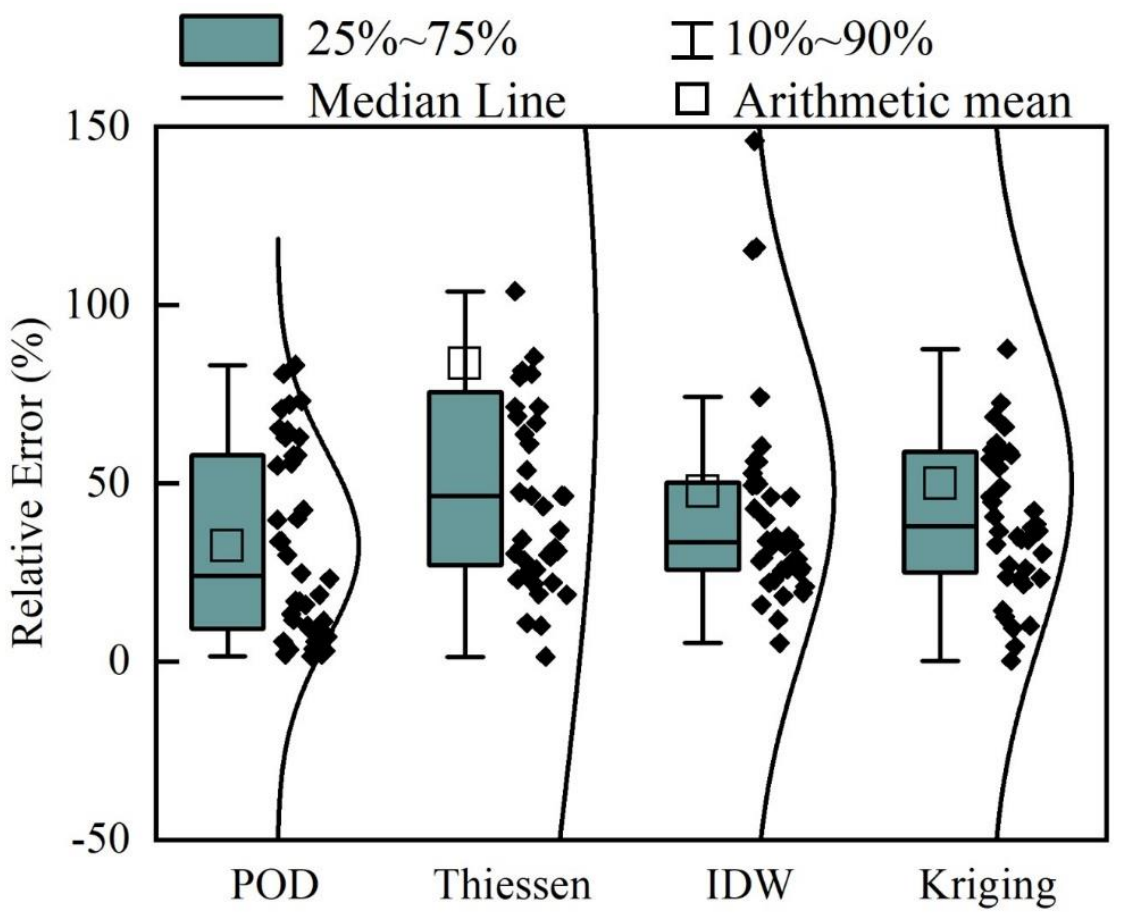

598 Fig. 11. Relative error box plots of the peak portion of simulated rainfall obtained by different methods. 

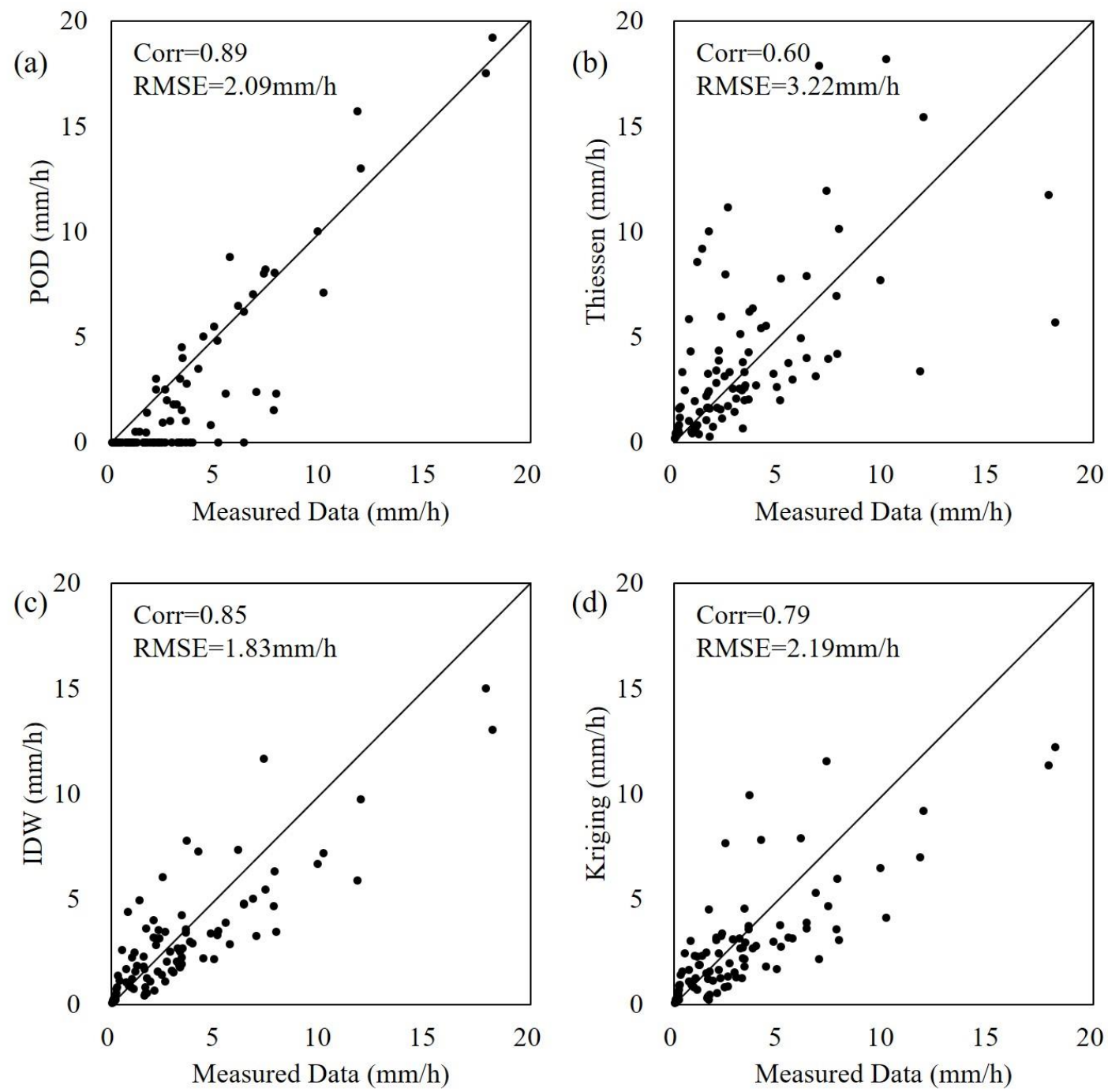

601

602 Fig. 12. Correlation diagram of rainfall obtained by different methods and measured 603 rainfall. 